\title{
Systemic signs of neutrophil mobilization during clinically stable periods and during exacerbations in smokers with obstructive pulmonary disease
}

This article was published in the following Dove Press journal:

International Journal of COPD

30 June 2015

Number of times this article has been viewed

\author{
Kristina Andelid' \\ Anders Andersson' \\ Shigemi Yoshihara ${ }^{2}$ \\ Christina Åhrén ${ }^{4}$ \\ Pernilla Jirholt ${ }^{3}$ \\ Ann Ekberg-Jansson' \\ Anders Lindén ${ }^{1,5}$ \\ 'Department of Internal Medicine \\ and Clinical Nutrition, Sahlgrenska \\ Academy at the University of \\ Gothenburg, Gothenburg, Sweden; \\ ${ }^{2}$ Department of Pediatrics, Dokkyo \\ Medical University, Japan; ${ }^{3}$ Department \\ of Rheumatology and Inflammation \\ Research, Institute of Medicine, \\ ${ }^{4}$ Department of Bacteriology, Institute \\ of Laboratory Medicine, Sahlgrenska \\ Academy at the University of \\ Gothenburg, Gothenburg; ${ }^{5}$ nnit \\ for Lung and Airway Research, \\ Institute of Environmental Medicine, \\ Karolinska Institutet, Stockholm, \\ Sweden
}

Correspondence: Anders Lindén Unit for Lung and Airway Research, Institute of Environmental Medicine, Karolinska Institutet, PO Box 210, SE-17I 77 Stockholm, Sweden

Tel +46700902286

Email anders.linden@ki.se
Background: It is still unclear whether signs of neutrophil mobilization in the blood of patients with chronic obstructive pulmonary disease represent true systemic events and how these relate to bacterial colonization in the airways. In this study, we evaluated these issues during clinically stable periods and during exacerbations in smokers with obstructive pulmonary disease and chronic bronchitis (OPD-CB).

Methods: Over a period of 60 weeks for each subject, blood samples were repeatedly collected from 60 smokers with OPD-CB during clinically stable periods, as well as during and after exacerbations. Myeloperoxidase (MPO) and neutrophil elastase (NE) protein and mRNA, growth of bacteria in sputum, and clinical parameters were analyzed. Ten asymptomatic smokers and ten never-smokers were included as controls.

Results: We found that, during clinically stable periods, neutrophil and NE protein concentrations were increased in smokers with OPD-CB and in the asymptomatic smokers when compared with never-smokers. During exacerbations, neutrophil and MPO protein concentrations were further increased in smokers with OPD-CB, without a detectable increase in the corresponding mRNA during exacerbations. However, MPO and NE protein and mRNA displayed positive correlations. During exacerbations, only increased neutrophil concentrations were associated with growth of bacteria in sputum. Among patients with low transcutaneous oxygen saturation during exacerbations, $\mathrm{PaO}_{2}$ (partial oxygen pressure) correlated with concentrations of MPO and NE protein and neutrophils in a negative manner.

Conclusion: There are signs of systemic neutrophil mobilization during clinically stable periods and even more so during exacerbations in chronic obstructive pulmonary disease. In this condition, MPO and NE may share a cellular origin, but its location remains uncertain. Factors other than local bacteria, including hypoxemia, may be important for driving systemic signs of neutrophil mobilization.

Keywords: C-reactive protein, COPD, elastase, infection, myeloperoxidase, oxygen

\section{Introduction}

Chronic obstructive pulmonary disease (COPD) is widely accepted as being a disorder characterized by signs of local and systemic inflammation. ${ }^{1,2}$ Clinical studies have suggested that signs of neutrophil mobilization, including increased concentrations of neutrophils, neutrophil-promoting growth factors, chemokines, and neutrophilderived enzymes, are of mechanistic interest. Moreover, there is evidence suggesting that systemic signs of inflammation are linked to the clinical course and comorbidity of COPD. ${ }^{2,3}$ We believe that further investigation into the specific nature of systemic signs of neutrophil mobilization in COPD is required. 
It is established that exacerbations of COPD constitute a major clinical risk factor for future morbidity and mortality. ${ }^{4,5}$ It is also established that these exacerbations account for a substantial part of the health care costs associated with COPD. Still, surprisingly little is known about the exact nature of neutrophil mobilization during clinically stable periods and during exacerbations of COPD. Some studies indicate that blood neutrophil concentrations are further increased from already elevated levels during these exacerbations. ${ }^{6-8}$ However, the location of the neutrophils accounting for circulating neutrophil-derived enzymes remains uncertain, during both clinically stable periods and exacerbations. ${ }^{3}$

Myeloperoxidase (MPO) is a functionally important enzyme that contributes to the destroying of bacteria during activation of the host defense system. ${ }^{9,10}$ Production of MPO takes place in neutrophil and monocyte precursor cells in the bone marrow, but very little is known about its production at the systemic level in COPD. ${ }^{9,10}$ In fact, there are currently no publications on the transcription of MPO at the systemic level in smokers with COPD, either during clinically stable periods or during exacerbations. The same is true for neutrophil elastase (NE), a neutrophil-derived enzyme that degrades structural lung protein and potently induces gland secretion during activation of the host defense. ${ }^{11}$

Given that long-term smokers inhale more than 5,000 different compounds over decades while slowly developing lung disease, there is potential for toxic effects on transcription of MPO or NE in circulating leukocytes. ${ }^{12}$ Consistent with this, we found that long-term smokers without severe airway symptoms developed increasing concentrations of MPO protein in their blood during a 6-year observation period. ${ }^{13}$ It therefore seems possible that systemic signs of neutrophil mobilization can occur prior to the clinical manifestation of COPD, and other investigators have also implicated MPO in the pathogenesis of COPD. One interesting study demonstrated a negative correlation between MPO in blood on the one hand, and ventilatory lung function, gas diffusion capacity, and dyspnea on the other in current and former smokers with COPD during clinically stable periods. ${ }^{14}$ Clearly this is compatible with pathogenic involvement of MPO originating from neutrophils in the blood and is also compatible with "spillover" from neutrophils accumulated in the lung; the same may be true for compounds other than MPO originating from the same azurophilic neutrophil granules, such as NE. ${ }^{11}$

For the current study, we hypothesized that there are systemic signs of neutrophil mobilization in smokers with COPD, and that these signs originate from blood leukocytes during clinically stable periods and during exacerbations. We reasoned that if this was the case, mobilization of neutrophils should include production and release of the neutrophil activity markers MPO and NE in blood leukocytes in vivo. ${ }^{10,11} \mathrm{We}$ investigated this hypothesis in a controlled, cross-sectional, longitudinal study in 60 smokers with obstructive pulmonary disease and chronic bronchitis (OPD-CB). We conducted repeated sampling of venous blood during clinically stable periods as well as during and after exacerbations for a total period of 60 weeks. We also related systemic signs of neutrophil mobilization to growth of bacteria in representative sputum samples. Ten asymptomatic long-term smokers (AS) and ten healthy never-smokers (NS) were recruited as control subjects. In addition to quantifying neutrophils as well as protein and mRNA for MPO and NE in blood samples, we quantified C-reactive protein (CRP) in this compartment and used it as a reference marker of true systemic inflammation. ${ }^{15-17}$

\section{Materials and methods Study subjects}

Sixty current and long-term smokers ( $>10$ pack-years) with OPD-CB were recruited from the outpatient clinic in the Department of Respiratory Medicine at Sahlgrenska University Hospital, Gothenburg, Sweden, and by advertisements in local newspapers. The patients were included during a clinically stable period and after denying respiratory tract infections in the 4 preceding weeks. We also included ten current and long-term AS (>10 pack-years) and ten NS with verified normal lung function as controls.

\section{Ethical considerations}

The study was approved by the ethics committee for medical research at the University of Gothenburg (S 233-03, $\mathrm{T}$ 286-04, and T 521-06). The study subjects were included after informed consent.

\section{Study protocol}

The subjects in this prospective, cross-sectional, longitudinal study were first examined during clinically stable periods at an inclusion visit (visit 1). At this visit, we critically evaluated the results of lung function tests (spirometry, gas diffusion capacity), pulmonary X-ray, physical examination, and blood, sputum, and urine samples. Blood samples were collected from the smokers with OPD-CB every 15 th week for the following 60 weeks (visits 2-5). If a patient reported signs of an exacerbation, an extra visit was arranged for additional blood and sputum sampling plus medical examination, after which regular medical treatment was given. All infections in the lungs/airways were deemed to 
be exacerbations. All infections in the airways or lungs were deemed to be exacerbations. No levels of CRP were utilized as exclusion criteria.

\section{Blood samples}

Plasma and serum samples were prepared for assessment of differential leukocyte counts and signs of neutrophil activity. The concentration of MPO protein $(\mu \mathrm{g} / \mathrm{mL})$ in plasma was measured using enzyme-linked immunosorbent assay. The concentration of NE protein $(\mu \mathrm{g} / \mathrm{mL})$ was measured using the latex bead concentration method. ${ }^{18,19}$ Quantitative polymerase chain reaction analysis of mRNA for MPO, NE, and carboxyl esterase (CES, a monocyte/macrophage marker) in blood samples from smokers with OPD-CB who developed exacerbations was conducted as described in detail in the Supplementary materials section.

\section{Sputum samples}

All smokers with OPD-CB provided sputum samples during a clinically stable period at the time of inclusion and during exacerbations. These samples were used for culture of bacteria, after we had ascertained that they were morphologically representative of the peripheral airways.

\section{Statistical methods}

Non-parametric descriptive and analytical statistics were used throughout this study with the help of Statistical Package for the Social Sciences version 16.0 software (SPSS Inc, Chicago, IL, USA) as appropriate. A $P$-value $<0.05$ was regarded as being statistically significant.

\section{Results \\ Clinically stable periods}

\section{Study population}

The clinical characteristics of the study subjects during clinically stable periods at the time of inclusion are published elsewhere. ${ }^{20}$ Briefly, smokers with OPD-CB of any Global Initiative for Chronic Obstructive Lung Disease stage were included, with only slight differences in age between the groups. Although we aimed to recruit equal numbers of men and women, there was a predominance of women in all study groups, and the tobacco load was somewhat higher in the group of smokers with OPD-CB than in the AS group. ${ }^{20}$

\section{Neutrophils and markers of neutrophil activity}

At the time of inclusion, neutrophil concentrations were clearly higher in smokers with OPD-CB and the AS group when compared with the NS group (Figure 1A), even though the differences were modest. However, there was no statistically significant difference in MPO protein concentrations between smokers with OPD-CB and the AS or NS groups; only weak trends toward an increase in smokers with OPD-CB were observed (Figure 1B). In contrast, NE protein concentrations were clearly higher in smokers with OPD-CB and in the AS group when compared with the NS group (Figure 1C). CRP concentrations were clearly higher in smokers with OPD-CB than in either of the control groups, ie, AS and NS (Figure 1D). In addition, there was a moderate positive correlation between MPO and NE protein concentrations and neutrophil concentrations in smokers with OPD-CB (Figure 2A and B). We were unable to identify a statistically significant correlation between CRP and NE ( $\rho=0.043, P>0.05$, Spearman rank correlation; $n=58)$ or between CRP and MPO $(\rho=0.16, P>0.05$, Spearman rank correlation; $n=59$ ) during clinically stable periods in smokers with OPD-CB at the time of inclusion (data not shown).

\section{Messenger RNA for MPO, NE, and CES in blood leukocytes}

At the time of inclusion, we detected a strong positive correlation between MPO and NE protein levels in all smokers with OPD-CB and for mRNA obtained from the 33 smokers with OPD-CB who subsequently developed exacerbations (Figure 3A and B). In addition, we detected a moderate correlation between mRNA for MPO and CES in smokers with OPD-CB ( $\rho=0.50, P<0.01$, Spearman rank correlation; $\mathrm{n}=32$, data not shown). There was no corresponding correlation detected between mRNA for CES and NE $(\rho=0.13, P>0.05$, Spearman rank correlation; $\mathrm{n}=32$ ) in smokers with OPD-CB (data not shown).

\section{Lung function and tobacco load}

At the time of inclusion, we failed to detect any linear correlations between MPO, NE, and CRP on the one hand and ventilatory lung function, gas diffusion capacity, and tobacco load on the other in the pooled group of smokers, ie, AS plus smokers with OPD-CB (data not shown).

\section{Sputum samples}

The results of sputum culture during clinically stable periods at the time of inclusion have been published elsewhere. ${ }^{20}$ Briefly, sputum samples for culture of bacteria were obtained from $87 \%$ of the smokers with OPD-CB at the time of inclusion, generating 40 samples that were representative of the peripheral airways. Seventeen of these representative samples grew common respiratory pathogens (mainly Haemophilus influenzae), 

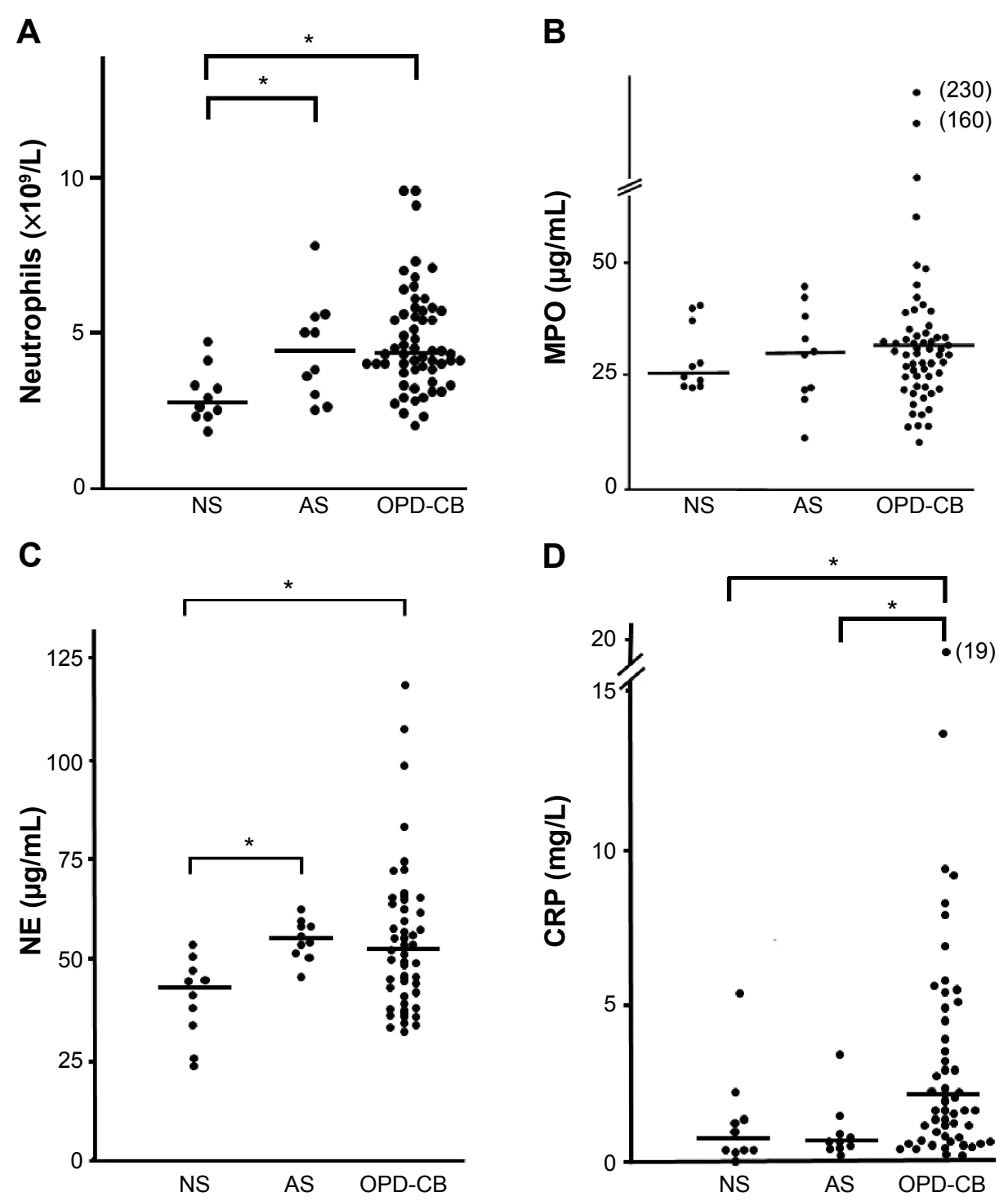

Figure I Blood concentrations of (A) neutrophils, (B) MPO protein, (C) NE protein, and (D) CRP in smokers with OPD-CB, AS, and NS during a clinically stable period at the time of inclusion.

Notes: The data are presented both as individual (dots) and median (bold lines) values $(* P<0.05$, Kruskal-Wallis test followed by the Mann-Whitney $U$-test, $n=10$ [neversmokers and asymptomatic smokers], $n=60$ [OPD-CB]).

Abbreviations: AS, asymptomatic smokers; CRP, C-reactive protein; MPO, myeloperoxidase; NE, neutrophil elastase; NS, never-smokers; OPD-CB, obstructive pulmonary disease and chronic bronchitis.

A

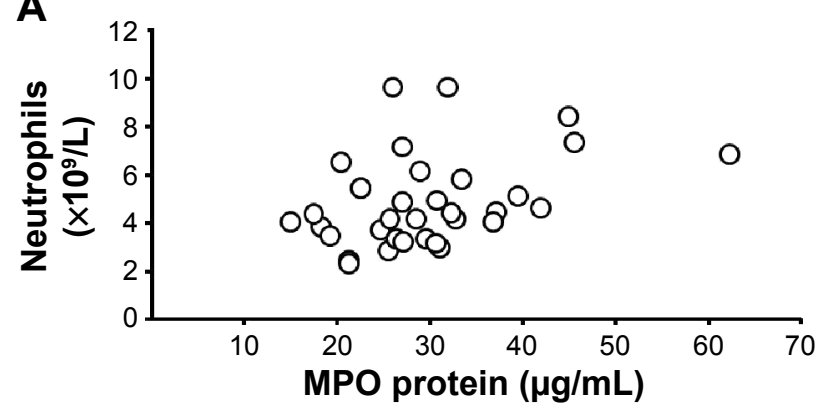

B

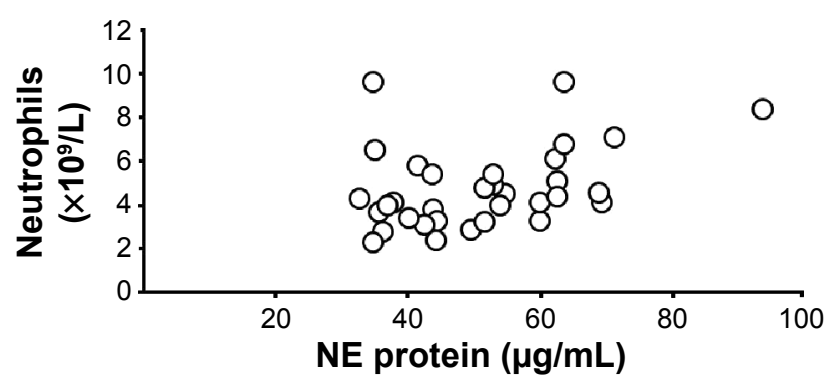

Figure 2 Correlations between (A) blood concentrations of MPO protein and neutrophils $(\rho=0.38, P<0.05$, Spearman rank correlation) and (B) blood concentrations of NE protein and neutrophils $(\rho=0.38, P<0.05$, Spearman rank correlation) of smokers with obstructive pulmonary disease and chronic bronchitis ( $n=34$ ) during a clinically stable period at the time of inclusion.

Abbreviations: MPO, myeloperoxidase; NE, neutrophil elastase. 
A

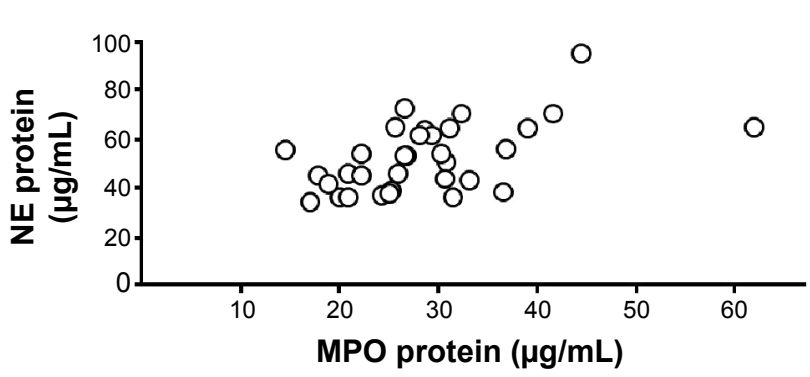

B

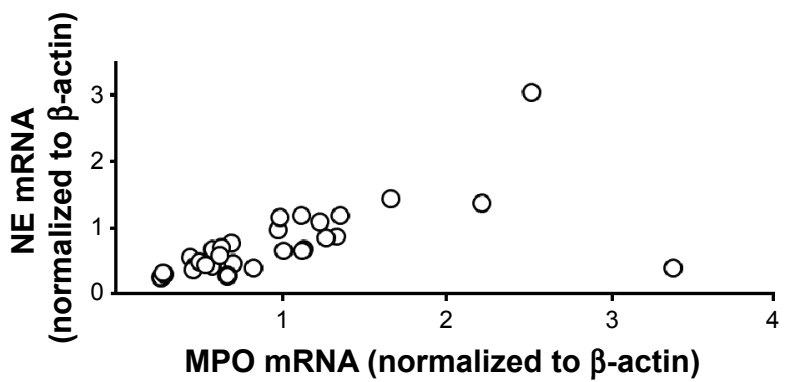

Figure 3 Correlations between (A) blood concentrations of MPO and NE protein ( $\rho=0.72, P<0.00$ I, Spearman rank correlation) and (B) levels of mRNA for MPO and $N E$ in blood leukocytes ( $r=0.85, P<0.00$ I, Spearman rank correlation) in smokers with obstructive pulmonary disease and chronic bronchitis ( $n=34$ ) during a clinically stable period at the time of inclusion.

Abbreviations: MPO, myeloperoxidase; NE, neutrophil elastase; OPD-CB, obstructive pulmonary disease and chronic bronchitis.

three grew opportunistic pathogens, and 20 grew oropharyngeal flora. Of note, there were no statistically significant differences ( $P>0.05$, Mann-Whitney $U$-test; $\mathrm{n}=40$ for all comparisons) in concentrations of MPO, NE, CRP, or neutrophils in smokers with OPD-CB who had a positive culture for common respiratory pathogens or oropharyngeal flora or a positive culture for opportunistic pathogens (data not shown). Furthermore, we did not find any statistically significant difference $(P>0.05$, MannWhitney $U$-test; $\mathrm{n}=40$ for all comparisons) in concentrations of MPO, NE, CRP, or neutrophils for smokers with OPD-CB who had, on the one hand, positive culture to either of the two types of pathogens and, on the other hand, those who had positive culture for oropharyngeal flora (data not shown).

\section{Exacerbations}

\section{Frequency}

Thirty-eight (63\%) smokers with OPD-CB had at least one exacerbation during the 60 -week study period. Of these, nine had a second and seven had a third exacerbation. For reasons to do with statistical power, we conducted our analysis using data from first exacerbation in each smoker with OPD-CB.

\section{Leukocytes, neutrophils, and markers of neutrophil activity}

Smokers with OPD-CB showed a clear but modest increase in leukocyte and neutrophil concentrations during exacerbations (Table 1). Likewise, the MPO protein concentration were increased in a clear but modest manner, and both neutrophils and MPO (Figure 4) tended to normalize at the time of the first regular visit after exacerbation, which was up to 15 weeks later. Moreover, NE protein tended to be increased during exacerbations but not in a statistically significant manner, whereas CRP was clearly increased during exacerbations (Table 1). Importantly, there was a correlation between concentrations of MPO and those of NE protein in smokers with OPD-CB during exacerbations (Figure 5A). In addition, smokers with OPD-CB showed a clear increase in CRP during exacerbations (Table 2). However, we failed to detect any correlation between values at the time of inclusion and those during exacerbations for MPO protein $(\rho=0.056$, $P>0.05$, Spearman rank correlation; $\mathrm{n}=35)$ or NE protein ( $\rho=0.053, P>0.05$, Spearman rank correlation; $n=36)$ and neutrophil $(\rho=0.009, P>0.05$, Spearman rank correlation; $\mathrm{n}=37$ ) concentrations, respectively; this was also true for CRP concentrations $(\rho=0.16, P>0.05$, Spearman rank correlation; $n=37$; data not shown). In addition to this, we did not find any predictive value of MPO or NE with regard to exacerbations (data not shown).

In terms of the relative exacerbation value for each sign of neutrophil mobilization or CRP("percent baseline", ie, the exacerbation value in relation to the value during clinically stable periods), the most pronounced change was in CRP (Figure S1). Moreover, the exacerbation-associated change (exacerbation value minus value during a clinically stable period) showed a correlation for protein concentrations of MPO and NE $(\rho=0.60, P<0.001$, Spearman rank correlation; $\mathrm{n}=33)$, MPO and CRP $(\rho=0.53, P<0.01$, Spearman rank correlation;

Table I Circulating markers of inflammation in smokers with OPD-CB

\begin{tabular}{llll}
\hline Marker & $\begin{array}{l}\text { Inclusion } \\
(\mathbf{n}=6 \mathbf{0})\end{array}$ & $\begin{array}{l}\text { Exacerbation } \\
\mathbf{( n = 3 8 )}\end{array}$ & P-value \\
\hline MPO $(\mu \mathrm{g} / \mathrm{mL})$ & $27(15-62)$ & $38(16-570)$ & $<0.0 \mathrm{I}$ \\
$\mathrm{NE}(\mu \mathrm{g} / \mathrm{mL})$ & $44(13 \mathrm{I}-102)$ & $53(23-2 \mathrm{I} 2)$ & $>0.05$ \\
$\mathrm{CRP}(\mathrm{mg} / \mathrm{L})$ & $2.3(2.0-19.0)$ & $6.5(0.3-300)$ & $<0.00 \mathrm{I}$ \\
Leukocytes $\left(\times 10^{9} / \mathrm{L}\right)$ & $7.1(4.5-13.5)$ & $8.3(5.4-16.0)$ & $<0.0 \mathrm{I}$ \\
Neutrophils $\left(\times 10^{9} / \mathrm{L}\right)$ & $4.1(2.3-9.6)$ & $5.5(3.1-13.0)$ & $<0.0 \mathrm{I}$ \\
\hline
\end{tabular}

Abbreviations: CRP, C-reactive protein; MPO, myeloperoxidase; NE, neutrophil elastase; OPD-CB, obstructive pulmonary disease and chronic bronchitis. 


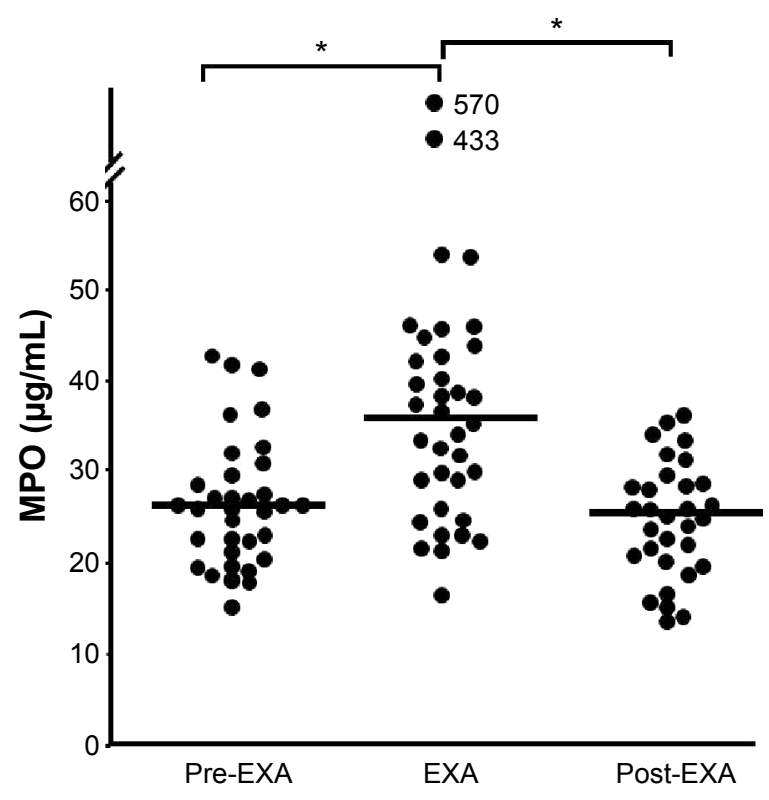

Figure 4 Blood concentrations of MPO protein before, during, and after exacerbations in smokers with obstructive pulmonary disease and chronic bronchitis $(n=38)$. Notes: The data are presented as individual (circles) and median (bold lines) values (*) $P<0.05$; Mann Whitney U-test).

Abbreviations: EXA, exacerbation; MPO, myeloperoxidase.

$\mathrm{n}=33)$, and NE and CRP $(\rho=0.68, P<0.001$, Spearman rank correlation; $\mathrm{n}=34$ ) in our cohort (data not shown).

mRNA for MPO, NE, and CES in leukocytes

There was no statistically significant $(P>0.05$, Wilcoxon signed rank test; $\mathrm{n}=33$ ) increase in MPO mRNA in the smokers with OPD-CB who developed exacerbations (data not shown). However, there was a strong correlation between mRNA for MPO and NE in these smokers during exacerbations (Figure 5B). The correlation between levels of mRNA for MPO and CES was weak $(\rho=0.35, P<0.05$, Spearman rank correlation; $\mathrm{n}=34$ ) among smokers with OPD-CB (data not shown) and there was no correlation between CES and mRNA for NE (data not shown). Moreover, there was no correlation ( $\rho=0.17, P<0.05$, Spearman rank correlation; $\mathrm{n}=33$ ) between the level of MPO mRNA during exacerbations and during clinically stable periods at the time of inclusion (data not shown).

\section{Arterial blood gas}

In 18 smokers with OPD-CB who developed exacerbations, the transcutaneous oxygen saturation was below $94 \%$, but none of these individuals required mechanical ventilation. In this respect, the clinical characteristics did not markedly differ for mild or severe OPD-CB (Table 2). Arterial blood gas was analyzed in all smokers with low transcutaneous oxygen saturation in accordance with clinical guidelines. We then detected statistically significant negative correlations between $\mathrm{PaO}_{2}$ (partial oxygen pressure) and concentrations of neutrophils and NE and MPO protein, respectively (Figure 6A-C). However, all smokers with OPD-CB had normal $\mathrm{PaCO}_{2}$ during exacerbations.

\section{Sputum samples}

Just like the case for clinically stable periods, bacteriology results from sputum culture during exacerbations have been published elsewhere. ${ }^{20}$ In summary, sputum samples were obtained from $46 \%$ of the smokers with OPD-CB during exacerbations, generating 29 samples representative of the peripheral airways. Of these, 14 samples grew common respiratory pathogens, none grew opportunistic pathogens, and 15 grew oropharyngeal flora. There was no statistically significant difference $(P>0.05$, Mann-Whitney $U$-test; $n=14-15)$ in the concentrations of MPO, NE, or CRP in smokers with OPD-CB who grew common respiratory pathogens and those who grew oropharyngeal flora (data not shown). However, there was a moderate $(P<0.05$, Mann-Whitney $U$-test; $\mathrm{n}=14-15)$ increase in neutrophil concentrations for patients with OPD-CB who grew common respiratory pathogens
A

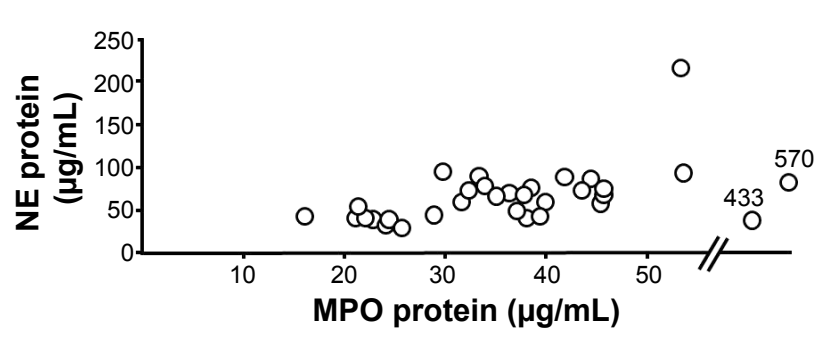

B

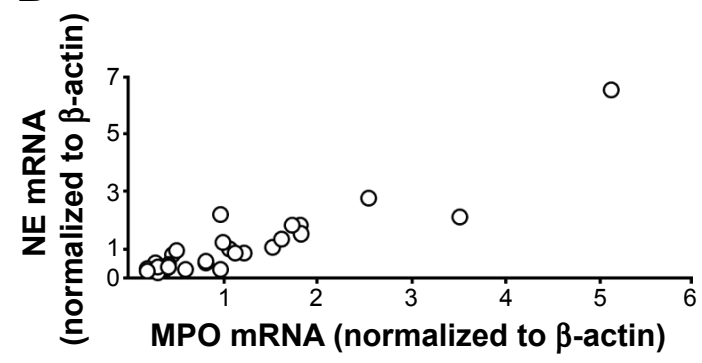

Figure 5 Correlations between (A) blood concentrations of MPO and NE protein $(\rho=0.4, P<0.05$, Spearman rank correlation) and (B) levels of MPO and NE mRNA in blood leukocytes $(\rho=0.89, P<0.00$ I, Spearman rank correlation) from smokers with obstructive pulmonary disease and chronic bronchitis ( $\mathrm{n}=34)$ during exacerbations. The data are presented as individual values.

Abbreviations: MPO, myeloperoxidase; NE, neutrophil elastase. 
Table 2 Characteristics of smokers with OPD-CB and low transcutaneous oxygen during exacerbations

\begin{tabular}{|c|c|c|c|c|c|c|}
\hline \multirow{2}{*}{$\begin{array}{l}\text { Severity of } \\
\text { OPD-CB }\end{array}$} & \multicolumn{3}{|l|}{ Inclusion $(n=18)$} & \multicolumn{3}{|c|}{ Exacerbation $(n=18)$} \\
\hline & $\begin{array}{l}\text { Transcutaneous oxygen } \\
\text { saturation (\%) }\end{array}$ & $\begin{array}{l}\text { Inhaled glucocorticoids } \\
\text { (yes/no) }\end{array}$ & $\begin{array}{l}\text { Respiration rate } \\
\text { (per minute) }\end{array}$ & $\mathrm{PaO}_{2}(\mathrm{kPa})$ & $\begin{array}{l}\text { Respiration rate } \\
\text { (per minute) }\end{array}$ & $\begin{array}{l}\text { Hospitalization } \\
\text { (yes/no) }\end{array}$ \\
\hline Mild $(n=10)$ & $95(94-98)$ & $6 / 4$ & $18(15-22)$ & $8.9(7.2-10.4)$ & $21(18-22)$ & $2 / 8$ \\
\hline Severe $(n=8)$ & $93(92-95)$ & $6 / 2$ & $20(16-24)$ & $7.8(7.3-9.8)$ & $20(16-26)$ & $\mathrm{I} / 7$ \\
\hline
\end{tabular}

Notes: All individuals in the table had transcutaneous oxygen saturation $<94 \%$ but none required mechanical ventilation during exacerbations; mild, OPD-CB corresponding to GOLD stage I and II; severe, OPD-CB corresponding to GOLD stage III-IV; the data are presented as the median and range ( $\mathrm{n}=8-\mathrm{I0}$ ).

Abbreviations: OPD-CB, obstructive pulmonary disease and chronic bronchitis; $\mathrm{PaO}_{2}$, partial oxygen pressure; GOLD Global Initiative for Chronic Obstructive Lung Disease.

(7.0 [3.9-13] 109/L) when compared with those who grew oropharyngeal flora $\left(5.2[3.1-10] 10^{9} / \mathrm{L}\right)$.

\section{Discussion}

During clinically stable periods, blood concentrations of neutrophils and NE protein but not MPO protein were increased in a modest manner in smokers with OPD-CB and in the AS control group when compared with the NS control group. However, there was no correlation between NE protein and mRNA, and the same was true for MPO during clinically stable periods in the smokers with OPD-CB. In contrast, we detected a strong positive correlation between MPO and NE protein during clinically stable periods; this was also true for MPO and NE mRNA. Taken together, these observations indicate involvement of neutrophils and NE in terms of mRNA levels and in terms of protein concentrations, during clinically stable periods in both AS and smokers with OPD-CB, with no detectable sign of de novo synthesis of NE in circulating blood leukocytes in the latter group. Moreover, the positive correlations between NE and MPO, both at the gene and the protein level, indicate that a shared cellular origin for these activity markers is very likely during clinically stable conditions.

During exacerbations in smokers with OPD-CB, blood concentrations of neutrophils and MPO protein, but not MPO
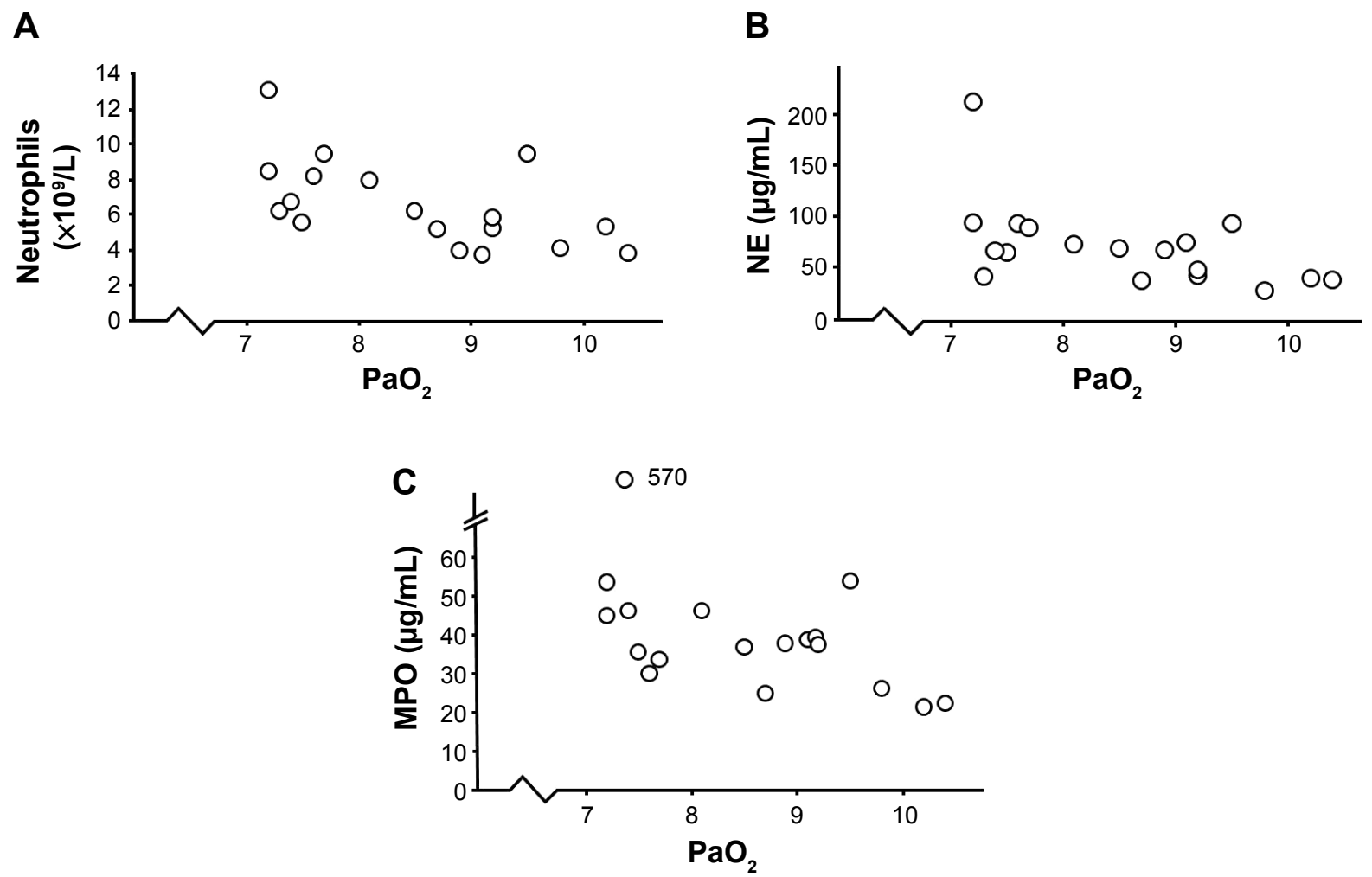

Figure 6 Correlations between oxygen saturation and signs of systemic inflammation in smokers with obstructive pulmonary disease and chronic bronchitis ( $\mathrm{n}=18$ ) who displayed transcutaneous hypoxia during exacerbations.

Notes: $(\mathbf{A}) \mathrm{PaO}_{2}$ and blood neutrophil concentrations ( $\rho=0.63, P<0.0 \mathrm{I}$, Spearman rank correlation). (B) $\mathrm{PaO}$ and blood concentrations of $\mathrm{NE}$ protein $(\rho=0.56, P<0.05$, Spearman rank correlation). (C) $\mathrm{PaO}_{2}$ and blood concentrations of MPO protein ( $\rho=0.5, P<0.05$, Spearman rank correlation).

Abbreviations: MPO, myeloperoxidase; $\mathrm{NE}$, neutrophil elastase; $\mathrm{PaO}_{2}$, partial oxygen pressure. 
mRNA, were increased in a clear but modest manner, and neutrophil and MPO concentrations tended to normalize after exacerbations, as assessed up to 15 weeks later. There was a matching trend toward an exacerbation-associated increase in NE protein, but not in NE mRNA. However, we detected no correlation between MPO mRNA and MPO protein in blood during exacerbations, and the same was true for NE. In contrast, we detected a strong positive correlation between MPO and NE, both in terms of mRNA and in terms of protein concentrations, during exacerbations. Taken together, these observations demonstrate the involvement of neutrophils and MPO during exacerbations in smokers with OPD-CB, although without any detectable signs of increased de novo synthesis of either NE or MPO in circulating blood leukocytes. Moreover, the positive correlations between NE and MPO, both at the gene and protein level, indicate that a shared cellular origin for these activity markers is very likely.

Growth of common respiratory pathogens in sputum was associated with increased neutrophil concentrations very likely, during exacerbations, but was neither associated with any substantial alteration in MPO, NE, or CRP concentrations in blood, nor during exacerbations or clinically stable periods. Further, growth of common bacterial pathogens was not associated with an increase in neutrophil concentrations during clinically stable periods. This implies that increased neutrophil concentrations can indicate bacterial infection during exacerbations in smokers with OPD-CB; however, in this sense, the clinical value of a corresponding increase in MPO and NE remains uncertain.

Both MPO and NE originate from azurophilic neutrophil granules ${ }^{9,11,21}$ which may be why these proteins were correlated and showed qualitatively similar changes in our current study, even though these changes were not consistently statistically significant. In line with this finding, we also detected a strong positive correlation between blood MPO and NE protein levels in a previous cohort study of malnourished patients with COPD during clinically stable periods. ${ }^{22}$

It is known that MPO may be present to a certain extent in circulating monocytes, in addition to being present in neutrophils. ${ }^{9}$ Thus, on hypothetical grounds, we think that the positive correlations found between MPO and CES mRNA levels during clinically stable periods at the time of inclusion and, even though weaker, during exacerbations, can be explained and are credible. The lack of a strong correlations between MPO and CES in our study seems compatible with a minor contribution from circulating monocytes. However, ex vivo studies of blood and airway cells are required for more definitive conclusions regarding the relative importance of the different putative cellular sources of MPO in patients with COPD.

We detected no increase in mRNA for MPO, NE, or CES in circulating blood leukocytes during exacerbations in smokers with OPD-CB. Further, there was a clear discrepancy between this negative finding and the demonstrated increase in MPO protein and neutrophil concentrations. There was also a trend toward increased NE protein concentrations during exacerbations, indicating a discrepancy between protein and mRNA for NE as well. Given these seemingly contradictory findings, it can be speculated that there is release of MPO and NE at the post-transcriptional level in circulating neutrophils during exacerbations, since this type of release may occur via exocytosis or neutrophil extracellular traps..$^{21,23}$ Likewise, it can be speculated that there is corresponding exocytosis or pathological transcription from neutrophils located in the extravascular compartment of the lung. ${ }^{3}$ These are interesting mechanistic possibilities that warrant further investigation in the future.

It is intriguing that several of our findings are compatible with the idea that smokers with OPD-CB suffer from neutrophil mobilization that is not merely driven by bacterial pathogens. We find it interesting that our current results are in line with those of a previous study suggesting that there is no association between the degree of upper or lower airway inflammation or bacterial load and systemic signs of inflammation. ${ }^{24}$ Also in line with previous studies, our current study demonstrated that bacterial growth was dominated by $H$. influenzae, a common respiratory pathogen. ${ }^{25,26}$ In addition, all the expected systemic signs of inflammation, including CRP, neutrophils and NE, were present. Therefore, we consider that our study population was reasonably representative of COPD.

In further support of factors other than bacteria driving systemic signs of inflammation, we detected a statistically significant negative correlation for concentrations of neutrophils, MPO, and NE versus $\mathrm{PaO}_{2}$ among the 18 smokers with OPD-CB who had transcutaneous oxygen saturation less than $94 \%$ during exacerbations. In fact, all targeted signs of neutrophil mobilization correlated with $\mathrm{PaO}_{2}$ in this subgroup in our current study cohort. We believe that this finding may have mechanistic implications in terms of understanding what drives neutrophilic inflammation in smokers with OPD-CB. This is because it has previously been demonstrated that experimentally induced hypoxemia in healthy human volunteers enhances the release of NE protein from circulating neutrophils. ${ }^{27}$ Thus, even though our current observation does not prove that hypoxemia, rather than exacerbations, actually drives systemic signs of neutrophil mobilization, it 
highlights the possibility that hypoxemia is an underestimated pathogenic factor in this context. ${ }^{28,29}$

In the pooled group of all smokers (AS and those with OPD-CB), there was a weak trend toward higher blood concentrations of MPO, one of the two investigated compounds originating from the azurophilic granules of neutrophils, during clinically stable periods. However, although this trend was not statistically significant, such a finding per se is compatible with the results of an earlier study reporting increased blood concentrations of MPO over several years in asymptomatic smokers. ${ }^{13}$ We think that the study population we selected might have contributed to our current results, but further study seems warranted in this respect. This is because levels of NE, the second of the two investigated compounds originating from azurophilic neutrophil granules, were clearly increased in AS and in smokers with OPD-CB during clinically stable periods in our current study. This latter observation raises the question of whether development of chronic bronchitis, a condition defined by hypersecretion in mucosal glands, is linked per se to a sustained systemic increase in the potent secretagogue NE. ${ }^{11,30}$ This observation also raises the question of whether the NE detected in blood represents spillover from the primary target organ for tobacco smoke (ie, the lungs).

We are not aware of any previous prospective, crosssectional, longitudinal studies in smokers with OPD-CB that have included repeated blood sampling during clinically stable periods as well as during exacerbations over 15 months or addressed signs of neutrophil mobilization at the systemic level. However, two previous studies have included data on concentrations of circulating MPO protein and eosinophil cationic protein (ECP) in stable COPD and chronic bronchitis. In one of these studies, the investigators demonstrated increased MPO and ECP protein concentrations in serum from patients with stable COPD. ${ }^{31}$ In the other study, ${ }^{32}$ the concentrations of MPO and ECP protein in serum were increased in COPD during exacerbations when compared with controls who did not suffer from COPD. ${ }^{32}$ Thus, our current findings are compatible with those of previously published studies; however, neither of the above-mentioned studies included data on systemic signs of neutrophil mobilization after remission of exacerbation. Therefore, the results of our current study both expand and confirm previous knowledge.

We also sought associations of an indisputable sign of systemic inflammation by assessing concentrations of CRP in blood. We tried to correlate these CRP concentrations with those of neutrophils, as well as with MPO and NE proteins. Here, we did note that the relative exacerbation-induced increase in CRP clearly exceeded that of MPO protein; a finding in line with that of a recently published study comparing 36 plasma biomarkers in COPD patients. ${ }^{17}$ The authors of that study claimed that CRP was the most "practically" useful of all the biomarkers in terms of confirming COPD exacerbations, in line with our current study results. Interestingly, in our study, we did not detect any sign of a correlation between CRP and MPO or NE protein concentrations in smokers with OPD-CB, and CRP was the only investigated marker of inflammation that was higher in smokers with OPD-CB than in the AS control group at the time of inclusion. However, in the smokers with OPD-CB, the exacerbation-associated change in CRP, MPO, and NE protein concentrations were correlated. Because of these findings, we speculate that CRP in reality reflects other aspects of inflammation than do signs of neutrophilic mobilization during clinically stable periods in COPD. In contrast, during exacerbations, CRP and signs of neutrophil mobilization may relate to one another, even though it remains unclear whether these signs of neutrophil mobilization are truly systemic or reflect spillover from local events in the lungs. ${ }^{3}$ This proposal is compatible with the results of several previous studies. . $^{14,33,34}$

Female subjects were over-represented in this study, so we cannot exclude the possibility of sex bias in our cohort. However, such bias is unlikely to explain our group-related differences since female over-representation was present in all our study groups. Moreover, we cannot exclude the possibility that our study population included some smokers with reversible airway obstruction (and this is why we used the term OPD rather than COPD). However, all included smokers denied a history of asthma or allergy, and tests for sensitization were negative when the study subjects were in doubt about allergy and should have excluded conditions other than COPD.

In conclusion, this study indicates clear systemic signs of neutrophil mobilization in COPD, that some of these signs are present in AS as well, and that the exact pattern of these signs may differ during clinically stable periods and during exacerbations. Moreover, this study suggests that there is no matching increase in transcription of MPO and NE in blood leukocytes in COPD, arguing against de novo synthesis of these activity markers in neutrophils circulating at the systemic level. The correlations between MPO and NE protein and mRNA are compatible with a common cellular origin, but the design of this study did not allow us to determine whether this origin is truly systemic (ie, extrapulmonary) or represents spillover from local inflammation (in the lungs). Except for neutrophil concentrations, 
the targeted systemic signs of neutrophil mobilization do not seem to be strongly associated with local bacterial growth. During exacerbations, hypoxemia and mobilization of neutrophils, irrespective of the location of the neutrophils, may be mechanistically linked in COPD. We think that further investigation of neutrophils isolated from various organs of patients with COPD is warranted to better understand the degree of true systemic involvement for these innate effector cells and the therapeutic implications thereof in COPD.

\section{Acknowledgments}

The current study cohort was also used for another recently published study. ${ }^{20}$ Thus, some data sets on clinical characteristics, bacteriology, and cell differential counts have been used for analysis in two separate publications. The authors would like to thank Barbro Balder for excellent study coordination and Johan Bylund, Department of Rheumatology and Inflammation Research, the Institute of Medicine, Sahlgrenska Academy at the University of Gothenburg, for advice regarding neutrophil biology. The authors also gratefully acknowledge the expert advice of Ingemar Qvarfordt, Department of Internal Medicine and Clinical Nutrition, Institute of Medicine, Sahlgrenska Academy, University of Gothenburg, regarding bacterial growth in sputum samples. Likewise, the authors gratefully acknowledge the scientific advice and technical support provided by Pernilla Glader and Ingrid Gjertsson, who were working at the Department of Internal Medicine and Clinical Nutrition and at the Department of Rheumatology and Inflammation Research, respectively, at the time of the study.

The study was supported financially by the Swedish Heart-Lung Fund (project 20130294), the Research Council (project K2008-57X-09048-19-3), federal funding within the ALF/LUA agreement for the Västra Götaland Region from King Gustaf V's and Queen Victoria's Freemason Foundation, and federal funding from the Karolinska Institutet. The authors gratefully acknowledge the funding for Kristina Andelid that was provided by Angered Local Hospital. No support was obtained from the tobacco industry.

\section{Disclosure}

The authors report no conflicts of interest in this work.

\section{References}

1. Global strategy for the diagnosis, management, and prevention of chronic obstructive pulmonary disease, 2012. Available from: www.goldcopd.com

2. Barnes PJ, Celli BR. Systemic manifestations and comorbidities of COPD. Eur Respir J. 2009;33(5):1165-1185.
3. Sinden NJ, Stockley RA. Systemic inflammation and comorbidity in COPD: a result of 'overspill' of inflammatory mediators from the lungs? Review of the evidence. Thorax. 2010;65(10):930-936.

4. Wedzicha JA, Donaldson GC. Exacerbations of chronic obstructive pulmonary disease. Respir Care. 2003;48(12):1204-1213.

5. Aburto M, Esteban C, Moraza FJ, Aguirre U, Egurrola M, Capelastegui A. COPD exacerbation: mortality prognosis factors in a respiratory care unit. Arch Bronconeumol. 2011;47(2):79-84.

6. de Jong JW, van der Belt-Gritter B, Koëter GH, Postma DS. Peripheral blood lymphocyte cell subsets in subjects with chronic obstructive pulmonary disease: association with smoking, IgE and lung function. Respir Med. 1997;91(2):67-76.

7. Wouters EF. Local and systemic inflammation in chronic obstructive pulmonary disease. Proc Am Thorac Soc. 2005;2(1):26-33.

8. Noguera A, Batle S, Miralles C, et al. Enhanced neutrophil response in chronic obstructive pulmonary disease. Thorax. 2001;56(6): 432-437.

9. Klebanoff SJ. Myeloperoxidase: friend and foe. J Leukoc Biol. 2005; 77(5):598-625.

10. Hickey MJ. MPO and neutrophils: a magnetic attraction. Blood. 2011; 117(4):1103-1104.

11. Roghanian A, Sallenave JM. Neutrophil elastase (NE) and NE inhibitors: canonical and noncanonical functions in lung chronic inflammatory diseases (cystic fibrosis and chronic obstructive pulmonary disease). J Aerosol Med Pulm Drug Deliv. 2008;21(1):125-144.

12. Pauly JL, Paszkiewicz G. Cigarette smoke, bacteria, mold, microbial toxins, and chronic lung inflammation. J Oncol. 2011;2011: 819129.

13. Andelid K, Bake B, Rak S, Lindén A, Rosengren A, Ekberg-Jansson A. Myeloperoxidase as a marker of increasing systemic inflammation in smokers without severe airway symptoms. Respir Med. 2007;101(5):888-895.

14. Aaron SD, Vandemheen KL, Ramsay T, et al. Multi analyte profiling and variability of inflammatory markers in blood and induced sputum in patients with stable COPD. Respir Res. 2010;11:41.

15. Broekhuizen R, Wouters EF, Creutzberg EC, Schols AM. Raised CRP levels mark metabolic and functional impairment in advanced COPD. Thorax. 2006;61(1):17-22.

16. Bafadhel M, Clark TW, Reid C, et al. Procalcitonin and C reactive protein in hospitalised adult patients with community acquired pneumonia, exacerbation of asthma and chronic obstructive pulmonary disease. Chest. 2011;139(6):1410-1418.

17. Hurst JR, Donaldson GC, Perera WR, et al. Use of plasma biomarkers at exacerbation of chronic obstructive pulmonary disease. Am J Respir Crit Care Med. 2006;174(8):867-874.

18. Hafner G, EH, Drosdat H, Lotz J, Eherental W, Wurzburg U. Evaluation of a new assay for the determination of PMN elastase-alfa-1proteinase inhibitor complexes in EDTA and citrated plasma. Clin Lab. 1997;43(1-2):3-9.

19. Yoshihara S, Yamada Y, Abe T, Lindén A, Arisaka O. Association of epithelial damage and signs of neutrophil mobilization in the airways during acute exacerbations of paediatric asthma. Clin Exp Immunol. 2006;144(2):212-216.

20. Andelid K, Tengvall S, Andersson A, et al. Systemic cytokine signaling via IL-17 in smokers with obstructive pulmonary disease: a link to bacterial colonization? Int J Chron Obstruct Pulmon Dis. 2015;10: 689-702.

21. Metzler KD, Fuchs TA, Nauseef WM, et al. Myeloperoxidase is required for neutrophil extracellular trap formation: implications for innate immunity. Blood. 2011;117(3):953-959.

22. Larsson S, Nordenson A, Glader P, Yoshihara S, Lindén A, Slinde F. A gender difference in circulating neutrophils in malnourished patients with COPD. Int J Chron Obstruct Pulmon Dis. 2011;6:83-88.

23. Metzler KD, Goosmann C, Lubojemska A, Zychlinsky A, Papayannopoulos V. A myeloperoxidase-containing complex regulates neutrophil elastase release and actin dynamics during NETosis. Cell Rep. 2014;8(3):883-896. 
24. Hurst JR, Perera WR, Wilkinson TM, Donaldson GC, Wedzicha JA. Systemic and upper and lower airway inflammation at exacerbation of chronic obstructive pulmonary disease. Am J Respir Crit Care Med. 2006;173(1):71-78.

25. Sykes A, Mallia P, Johnston SL. Diagnosis of pathogens in exacerbations of chronic obstructive pulmonary disease. Proc Am Thorac Soc. 2007;4(8):642-646.

26. Tumkaya M, Atis S, Ozge C, Delialioglu N, Polat G, Kanik A. Relationship between airway colonization, inflammation and exacerbation frequency in COPD. Respir Med. 2007;101(4):729-737.

27. McGovern NN, Cowburn AS, Porter L, et al. Hypoxia selectively inhibits respiratory burst activity and killing of Staphylococcus aureus in human neutrophils. J Immunol. 2011;186(1):453-463.

28. Brusselle GG, Joos GF, Bracke KR. New insights into the immunology of chronic obstructive pulmonary disease. Lancet. 2011;378(9795): 1015-1026.
29. Baines KJ, Simpson JL, Gibson PG. Innate immune responses are increased in chronic obstructive pulmonary disease. PLoS One. 2011;6(3):e18426.

30. Hoenderdos K, Condliffe A. The neutrophil in chronic obstructive pulmonary disease. Am J Respir Cell Mol Biol. 2013;48(5):531-539.

31. Amici MD, Moratti R, Quaglini S, Alesina R, Peona V. Increased serum inflammatory markers as predictors of airway obstruction. J Asthma. 2006;43(8):593-596.

32. Fiorini G, Crespi S, Rinaldi M, Oberti E, Vigorelli R, Palmieri G. Serum ECP and MPO are increased during exacerbations of chronic bronchitis with airway obstruction. Biomed Pharmacother. 2000;54(5):274-278.

33. de Torres JP, Cordoba-Lanus E, López-Aguilar C, et al. C-reactive protein levels and clinically important predictive outcomes in stable COPD patients. Eur Respir J. 2006;27(5):902-907.

34. Pinto-Plata VM, Müllerova H, Toso JF, et al. C-reactive protein in patients with COPD, control smokers and non-smokers. Thorax. 2006; 61(1):23-28.

\section{Publish your work in this journal}

The International Journal of COPD is an international, peer-reviewed journal of therapeutics and pharmacology focusing on concise rapid reporting of clinical studies and reviews in COPD. Special focus is given to the pathophysiological processes underlying the disease, intervention programs, patient focused education, and self management protocols.

\section{Dovepress}

This journal is indexed on PubMed Central, MedLine and CAS. The manuscript management system is completely online and includes a very quick and fair peer-review system, which is all easy to use. Visit http://www.dovepress.com/testimonials.php to read real quotes from published authors.

Submit your manuscript here: http://www.dovepress.com/international-journal-of-chronic-obstructive-pulmonary-disease-journal 